\title{
sciendo
}

\section{THE SSC15 QTL-RICH REGION MUTATIONS AFFECTING INTRAMUSCULAR FAT AND PRODUCTION TRAITS IN PIGS*}

\author{
Katarzyna Piórkowska ${ }^{1 \star}$, Mirosław Tyra², Kacper Żukowski³ ${ }^{3}$, Katarzyna Ropka-Molik \\ ${ }^{1}$ Department of Animal Molecular Biology, \\ ${ }^{2}$ Department of Pig Breeding, \\ ${ }^{3}$ Department of Cattle Breeding, \\ National Research Institute of Animal Production, 32-083 Balice n. Kraków, Poland \\ •Corresponding author: katarzyna.piorkowska@izoo.krakow.pl
}

\begin{abstract}
One of the more interesting regions in the pig genome is on chromosome $15(115,800,000-$ $122,100,000$, SSC15, Sus scrofa 11.1) that has high quantitative trait locus (QTL) density associated with fattening, slaughter and meat quality characteristics. The SSC15 region encodes over 80 genes and a few miRNA sequences where potential genetic markers can be found. The goal of the study was to evaluate the effects of SSC15 mutations associated with villin 1 (VIL1), tensin 1 (TNS1), obscurin-like 1 (OBSL1) genes and with one long non-coding RNA (IncRNA) on productive pig traits and to enrich the genetic marker pool in further selection purpose. The potential genetic markers were identified using the targeted enrichment DNA sequencing (TEDNA-seq) of chromosome 15 region. The selected mutations were genotyped by using HRM, PCR and PCRRFLP methods. The association study was performed using the general linear model (GLM) in the SAS program that included over 600 pigs of 5 Polish populations. The rs332253419 VIL1 mutation shows a significant effect on intramuscular fat (IMF) content in Duroc population where AA pigs had a $16 \%$ higher level than heterozygotes. The IMF content is also affected by the $O B S L 1$ mutation, and the differences between groups are even up to $30 \%$, but it is strongly dependent on breed factor. The $O B S L 1$ mutation also significantly influences the meat yellowness, backfat thickness and $\mathrm{pH}$ level. The performed study delivers valuable information that could be highly useful during the development of the high-throughput genotyping method for further selection purposes in pigs. The $O B S L 1$ and $V I L 1$ mutations seem to be the most promising DNA marker showing a high effect on IMF level.
\end{abstract}

Key words: obscurin-like-1, villin, IncRNA, targeted DNA sequencing, pork quality

*The targeted enrichment DNA sequencing method and partly association study was funded by Research Project No. 2013/09/D/NZ9/02452 of Polish National Science Center and the rest of the analysis was funded by National Research Institute of Animal Production statutory activity "FBW program", research project No. 04-18-01-21. 
Over the decades, farm animal quantitative trait loci (QTL) were estimated in the numerous studies aiming to narrow the search area for genetic markers. The publicly available pig QTL database contains chromosome regions associated with utility traits and genes affecting phenotypic traits of interest.

One of the most interest chromosome regions in the porcine genome is a part of chromosome 15 (SSC15), which exhibits high QTL density (approx. 300 QTLs) - loci associated with fattening, slaughter and meat quality traits. This chromosome region encodes over 80 genes and a few miRNA sequences. It was previously sequenced using targeted enrichment strategy, during DNA sequencing (TEDNA-seq) based on next-generation sequencing technology which was described by Piórkowska et al. (2018). In the recent studies, numerous genes and miRNAs located in the SSC15 region were evaluated for their effects on pig production traits, such as 5'-AMPactivated protein kinase subunit gamma-3 (PRKAG3), E3 ubiquitin-protein ligase (MARCH4), ATP-binding cassette sub-family A member 12 (ABCA12), miR-4334, desmine $(D E S)$, miR-26, insulin-like growth factor binding protein 2 (IGFBP2), and peroxisomal trans-2-enoyl-CoA reductase (PECR). The $P R K A G 3$ encodes a subunit of 5'AMP-activated protein kinase (AMPK) that plays a crucial role in the energy metabolism of skeletal muscle and affects pork quality. Zhang et al. (2015) identified QTL close to porcine PRKAG3 affecting $\mathrm{pH}$ value and pork colour using genome wide association study (GWAS). Similar relations were found by Dall'Olio et al. (2018), who suggested including PRKAG3 in the porcine major gene list. In turn, Ponsuksili et al. (2017) analysing the effect of hepatic miRNAs found that miR-4334 has a highly positive impact on inorganic phosphorus (IP), which is key for the energy control by formation of adenosine triphosphate (ATP) from adenosine diphosphate (ADP) (Dodds et al., 2010). In another study, Ropka-Molik et al. (2015) indicated that single nucleotide polymorphisms (SNPs) in the MARCH4 gene affect ham and loin mass, and backfat thickness. Subsequently, Piórkowska et al. (2017) showed that PECR expression negatively correlates with backfat thickness and shows a high association with SNP in the promoter region of this gene. Moreover, the authors analysing $P E C R$ promotor region mutation revealed that it affects intramuscular fat (IMF) content, water holding capacity (WHC) and pork texture parameters.

Piórkowska et al. (2018) sequenced the QTL-rich SSC15 region and suggested that numerous mutations probably affect pig phenotype and can become genetic markers used in the selection procedure. In the present study, five mutations localised within, or in the neighbourhood of genes were evaluated for their effect on pig production traits using various molecular methods, including over 600 pigs that belonged to five different breeds; villin 1 (VIL1), which was also indicated by Zhang et al. (2015) that is located in locus associated with meat colour and $\mathrm{pH}$ value, obscurin-like 1 (OBSL1), transition protein 1 (TNP1), lncRNA, and tensin 1 (TNS1) that was found by Ji et al. (2019) to be associated with average daily gains in different age. 


\section{Material and methods}

\section{Animals}

The study included 663 pigs originating from different farms and belonging to five Polish pig breeds: Landrace ( $n=135$, PL), Large White $(n=236$, PLW), Pietrain $(n=81)$, Duroc $(n=58)$ and Puławska $(n=153$, PUL). The pigs had the unrelated pedigree and were of one gender (females). They were delivered from different farms to the Pig Test Stations (PTS-Chorzelów, Mełno, Pawłowice, and Rossocha) when they had $30 \mathrm{~kg}$ of body mass (BM), and they were maintained until reaching $100 \mathrm{~kg}$ of $\mathrm{BM}$ in the same housing and feeding conditions during the fattening period according to the PTS procedure (Różycki and Tyra, 2010). During fattening, the growth traits, such as daily gain (g/day) (DG), feed conversion ( $\mathrm{kg} / \mathrm{kg}$ ) (FC) and daily feed intake (kg) (DFI) were recorded. Afterwards, the pigs were slaughtered and the right halfcarcases chilled for $24 \mathrm{~h}$ at $4^{\circ} \mathrm{C}$. Subsequently, the right half-carcasses was measured and dissected to estimate carcass performance and meat quality as described by Tyra and Żak (2013), including loin (LM, kg) and ham (HM, kg) mass, average backfat thickness (cm) (ABT), loin eye area $\left(\mathrm{cm}^{2}\right)$ (LEA), carcass yield $(\%)$, meat colour, IMF content and $\mathrm{pH}$ in the longissimus dorsi muscle. $\mathrm{pH}$ was measured 45 minutes after the slaughter at last rib according to the PTS procedure, meat colour parameters (redness $-\mathrm{A}$, yellowness $-\mathrm{B}$, and lightness $-\mathrm{L}^{*}$ ) were estimated by colourimeter Minolta CR-300, intramuscular fat (IMF) content was assessed in thawed homogenates by the Soxhlet method using Soxtherm SOX 406 - Gerhardt (Soxhlet, 1879).

\section{DNA isolation}

DNA was isolated using Sherlock AX kit (A\&A Biotechnology, Gdańsk, Poland) from $100 \times 1$ EDTA stabilised blood samples collected after slaughter. The quality and quantity of DNA isolates were measured with a NanoDrop2000.

Targeted enrichment DNA sequencing and selection of potential biomarkers

The TEDNA-seq analysis was performed as described previously by Piórkowska et al., (2018) using RNA hybrid capture (1x tiling) designed by the Agilent team. The samples for TEDNA-seq were unique indexed, which made it possible to obtain a polymorphism profile for each sample individually and to carry out a preliminary association study using LRT test in R-project as described by Stuczyńska et al. (2018). The selection criteria of gene variants for further analysis were as follows: LRT test P-value $\leq 0.05$ for at least a few investigated productive pig traits, and a relationship of identified mutations with genes involved in the determination of pig phenotype based on functional analysis using Panther, STRING and KEGG. All selected gene variants were confirmed by Sanger sequencing using the BigDye ${ }^{\circledR}$ Terminator v3.1 Sequencing Kit (Applied Biosystems, USA) on a 3500XL Genetic Analyser (Applied Biosystems, USA).

\section{Genotyping of selected mutations with the potential to become biomarkers}

Based on functional analysis and the LRT results, five mutations were selected and tested in the association study, including PUL, PL, PLW, Pietrain and Duroc breeds. The pigs were genotyped by using different molecular techniques. 
rs332253419 (A/T) of VIL1 and rs344208061 C/T of TNS1 missense variants were genotyped by the PCR-RFLP method, AmpliTaq Gold ${ }^{\mathrm{TM}} 360$ master mix (Applied Biosystems, USA), and HpyCH4IV and AciI restriction enzymes, respectively. rs332398561 (C/A) of OBSL1 missense variant was analysed by the high-resolution melting (HRM) technique on a QuantStudio 7 Flex Real-Time PCR System (Applied Biosystems, USA) using KAPA ${ }^{\mathrm{TM}}$ HRM Fast PCR Kit (Kapa Biosystems, USA). rs711919547 and rs693217735 indel type mutations located upstream of TNP1 and in the intron of long non-coding RNA (lncRNA) (ENSSSCG00000033265) were genotyped by PCR technique and AmpliTaq Gold ${ }^{\mathrm{TM}} 360$ master mix (Applied Biosystems) by $55^{\circ} \mathrm{C} \mathrm{T}_{\mathrm{a}}$.

\section{Statistical analysis}

The GLM procedure (SAS v.8.02) calculated mutation effects for all pigs. The final model was

$$
Y_{i j k}=\mu+d_{i}+b_{j}+s_{k}+\alpha\left(x_{i j k}\right)+e_{i j k}
$$

where:

$Y_{i j k}$ - observation,

$\mu$ - overall mean of the trait,

$d_{i}$ - fixed effect of the $\mathrm{k}_{\text {th }}$ genotypes,

$b_{j}$ - fixed effect of breed,

$\mathrm{s}_{\mathrm{k}}$ - fixed effect of pig test station,

$\alpha\left(x_{i j k}\right)$ - covariance in weight of right carcass,

$e_{i j k}$-random error.

The model used to estimate the differences within each breed was $Y_{i j k}=\mu+d_{i}+s_{k}+e_{i j k}$. The differences between genotype groups were calculated by least square mean (LSM) test. All results are shown as LSM \pm SE. The Hardy-Weinberg Equilibrium (HWE) was measured using the HWE calculator (www.dr-petrek.eu/documents/ HWE.xls). If P-value was $<0.05$, results were regarded as not consistent with HWE. Transcription factors were predicted by PROMO3 software (Messeguer et al., 2002). miRNA binding motif was identified by regRNA2.0 (Chang et al., 2013). The potential miRNA coding sequence was identified by sequence-structure Motif Base: Pre-miRNA Prediction Webserver.

\section{Results}

\section{Animals}

The differences between analysed breeds were presented in Table S1. The highest differences in meat quality were observed between Duroc and Pietrain pigs. However, these breeds showed similar IMF content values and White pigs and Puławska were characterized by low IMF content. In turn, considering dissected traits, the highest extremes were observed in Pietrain and Puławska populations. 







\section{The selection of interesting gene variants}

Table 1 presents data for the investigated mutations (VIL1, OBSL1, TNP1, IncR$N A$ and TNS1). Table 2 shows the LRT test results.

Table 1. Chosen variation of genes that could be associated with pig production traits

\begin{tabular}{c|c|c|c|c|c}
\hline ID Number & $\begin{array}{c}\text { Position on } \\
\text { SSC15 }\end{array}$ & $\begin{array}{c}\text { Reference } \\
\text { allele }\end{array}$ & $\begin{array}{c}\text { Alternate } \\
\text { allele }\end{array}$ & $\begin{array}{c}\text { Localisation or } \\
\text { effect }\end{array}$ & Gene \\
\hline rs693217735 & $\begin{array}{c}119635224- \\
119635254\end{array}$ & $\begin{array}{c}\text { 31-bp-long } \\
\text { insertion }\end{array}$ & - & Intron variant & $\begin{array}{c}\text { ENSSSCG00000033265 } \\
\text { (lncRNA) }\end{array}$ \\
rs790770289 & $\begin{array}{c}119332342- \\
16-b p-l o n g\end{array}$ & - & Intergenic region & TNP1 - ENSSSCG00000040247 \\
insertion & & & & \\
rs332253419 & 119332357 & 120476956 & $\mathrm{~A}$ & $\mathrm{~T}$ & Missense variant VIL1 \\
rs344208061 & 119997161 & $\mathrm{C}$ & $\mathrm{T}$ & Missense variant ENSSSCG00000027669 (TNS1) \\
rs791506568 & 121561483 & $\mathrm{C}$ & $\mathrm{A}$ & Missense variant OBSL1 \\
\hline
\end{tabular}

Validation of significant gene variants by using the Sanger sequencing method Investigated mutations were confirmed by Sanger sequencing method and 3500XL Genetic Analyser. The obtained results are presented in Figure 1.


Figure 1. The chromatogram showing the variations of VIL1, OBSL1, TNS1, TNP1 and ENSSS$C G 00000033265$ mutations 


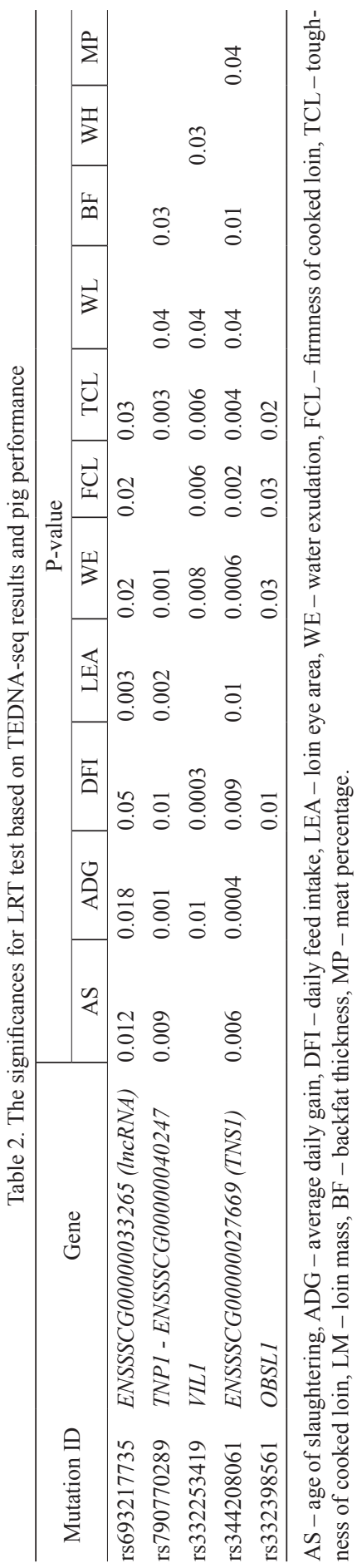




\section{The effect of mutations on pig phenotype}

\section{VIL1 missense variant}

The PCR-RFLP method was used for genotyping of rs332253419 VIL1 mutation. It changes $\mathrm{A} / \mathrm{T}$ in position 887 of VIL1 coding region that leads to replacing of tyrosine for phenylalanine in the amino acid sequence encoding important VIL1 domains. T allele occurred with 0.13 frequency, and TT genotype constituted only $9 \%$ in the investigated pig populations. TT pigs were the most numerous in the Pietrain population (21\%), and in the Duroc breed, this genotype was very rare (Table S1).

AA PLW pigs were the most frequent in this population and were characterised by the highest IMF level in comparison to the other pigs ( $\mathrm{P} \leq 0.05)$ (Table 3 ). Heterozygous AT Duroc pigs showed significantly increased IMF level in comparison to AA pigs ( $\mathrm{P} \leq 0.01)$. Moreover, in breeds used in the Polish breeding as maternal components (PLW and PL), AA pigs showed the highest carcass yield and in the native Puławska, the opposite effect was observed; TT pigs were characterised by increased ham mass and loin eye area (LEA) $\left(5.1 \mathrm{~cm}^{2}, \mathrm{P} \leq 0.01\right)$, and thicker backfat. Regarding fattening traits, the AA pigs showed the highest feed intake, but the differences within breeds were not significant due to the low number of individuals in particular groups.

\section{OBSL1 gene}

All investigated populations, except PL, were consistent with Hardy-Weinberg Equilibrium according to rs332398561(C/A) variation in the OBSL1. CC genotype dominated in the PLW, PUL, Duroc and Pietrain populations, and AA genotype in the PL breed. C $>$ A OBSL1 missense variant leads to exchange of alanine to serine at position 1785 of the amino acid sequence in the immunoglobin-like protein domain. Allele and genotype frequencies are shown in Table S2.

Table S2. The frequencies of alleles and genotypes of rs332253419 (A/T) in VIL1 gene in 5 Polish breeds

\begin{tabular}{|c|c|c|c|c|c|c|}
\hline \multirow{3}{*}{ Pig breed } & \multicolumn{6}{|c|}{ Frequencies of rs332253419(A/T) in VIL1 gene } \\
\hline & \multicolumn{3}{|c|}{ genotype } & \multicolumn{2}{|c|}{ allele } & \multirow{2}{*}{$\begin{array}{l}\text { HWE* } \\
\text { (P-value) }\end{array}$} \\
\hline & AA & AT & TT & A & $\mathrm{T}$ & \\
\hline Polish Large White & $0.53(120)$ & $0.39(88)$ & $0.08(17)$ & 0.72 & 0.28 & 0.87 \\
\hline Polish Landrace & $0.43(55)$ & $0.50(63)$ & $0.07(9)$ & 0.68 & 0.32 & 0.11 \\
\hline Puławska & $0.36(50)$ & $0.54(74)$ & $0.10(14)$ & 0.63 & 0.37 & 0.08 \\
\hline Duroc & $0.69(29)$ & $0.29(12)$ & $0.02(1)$ & 0.83 & 0.17 & 0.85 \\
\hline Pietrain & $0.28(19)$ & $0.51(34)$ & $0.21(14)$ & 0.53 & 0.47 & 0.87 \\
\hline Total & $0.46(273)$ & $0.45(271)$ & $0.09(55)$ & 0.87 & 0.13 & \\
\hline
\end{tabular}

*HWE - Hardy-Weinberg Equilibrium: if P-value $<0.05$ - not consistent with HWE; not accurate if $<5$ individuals in any genotype group. In brackets number of individuals in genotype group. 


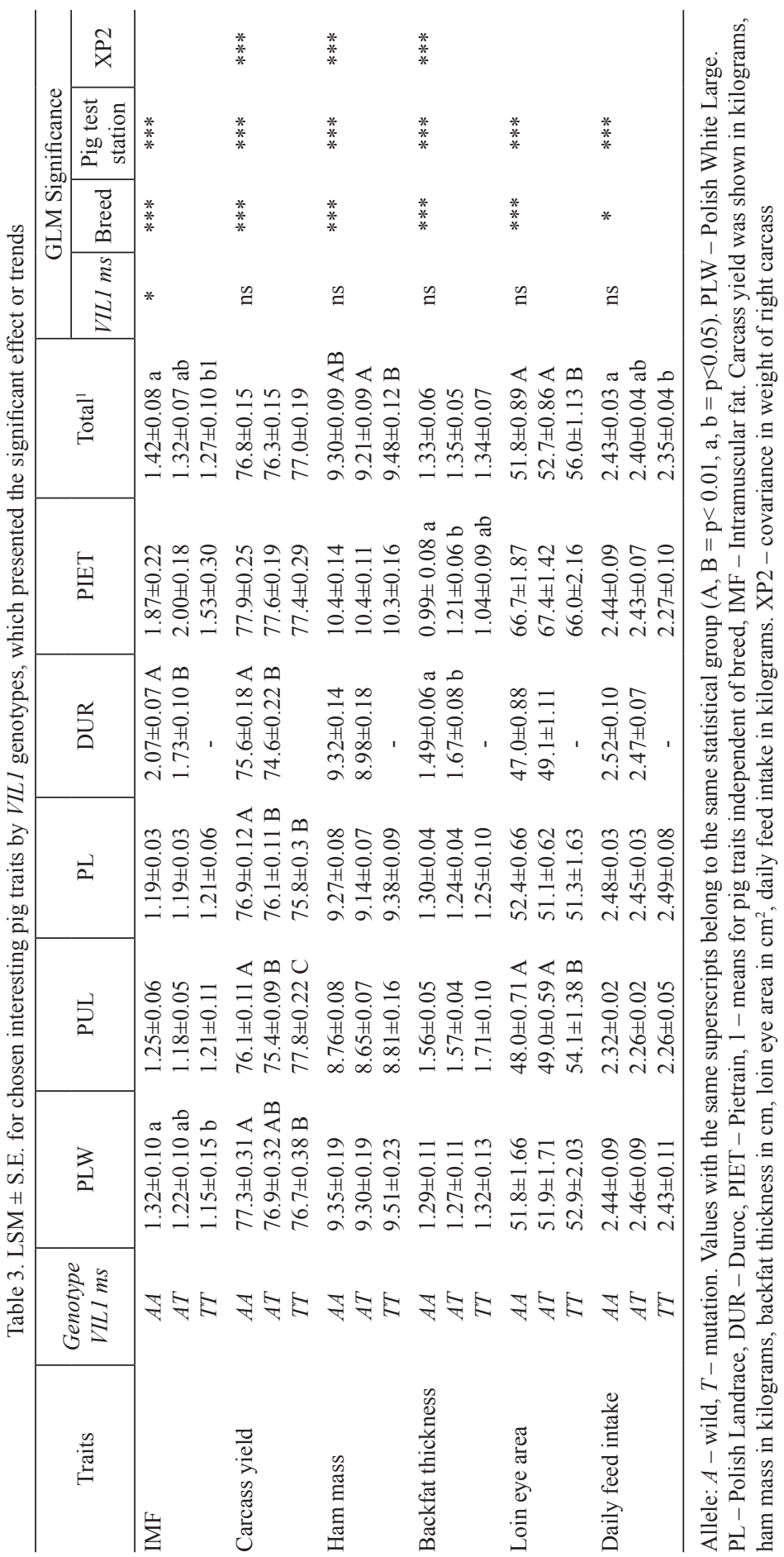




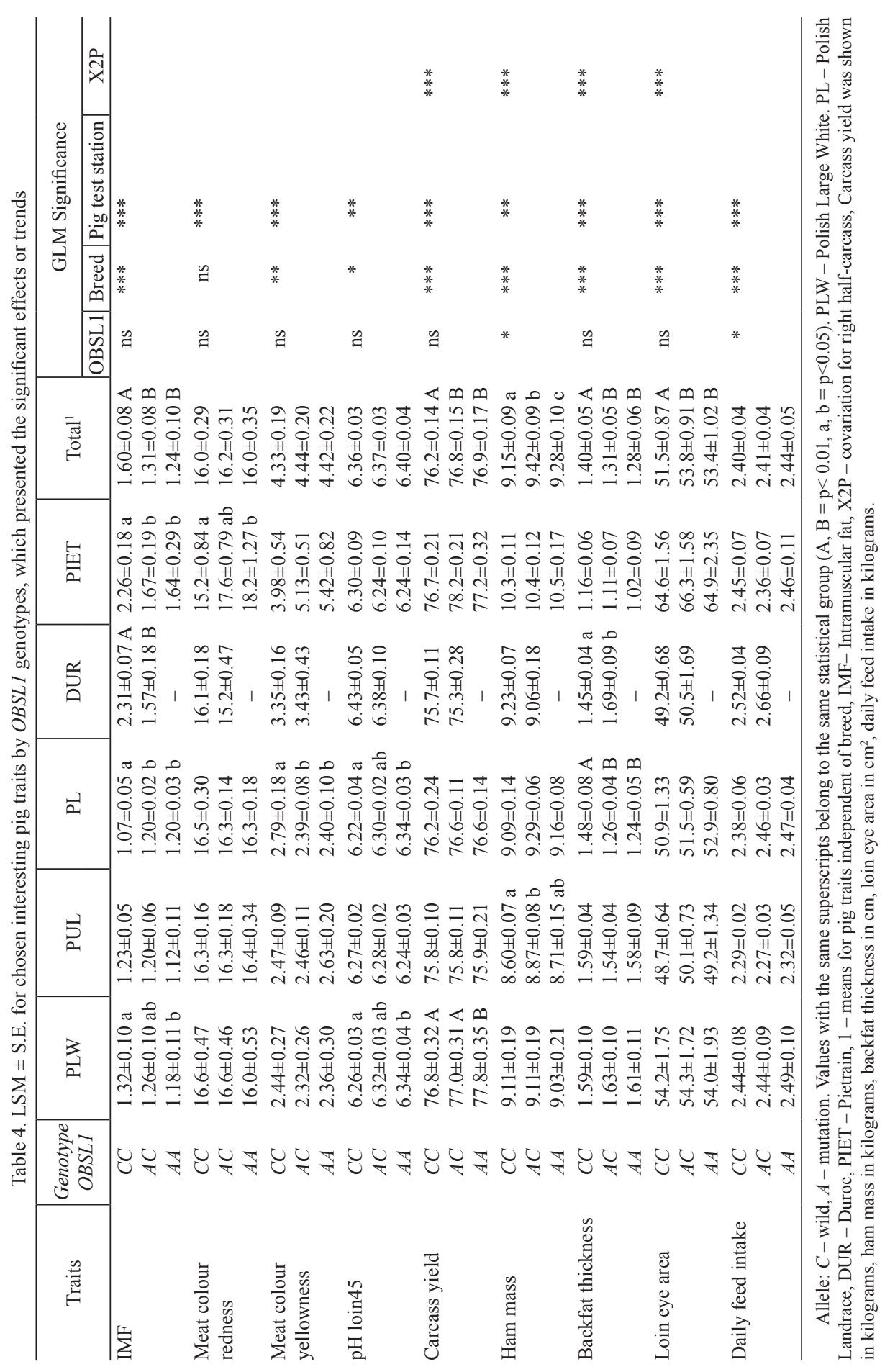


In Duroc pigs, where only one AA pig was identified, $\mathrm{CC}$ gilts had $31 \%$ higher IMF level in comparison to heterozygous CA pigs $(\mathrm{P} \leq 0.01)$. The difference in IMF level between AA and CC pigs was $26 \%(\mathrm{P} \leq 0.05)$ in the Pietrain and $11 \%$ in the PLW $(\mathrm{P} \leq 0.05)$. In the $\mathrm{PL}$ population the opposite trend was observed - $\mathrm{CC}$ pigs had the lowest IMF level $(\mathrm{P} \leq 0.05)$ and they were also characterised by higher meat yellowness, the lowest loin $\mathrm{pH}_{45}$ values, the thickest backfat $(\mathrm{P} \leq 0.01)$, the lowest loin eye area and daily feed intake (DFI) (trends) (Table 4).

\section{TNS1 gene}

rs344208061 C/T TNS1 missense variant is localised in the second exon of TNS1 gene non-coding any protein domains. The $\mathrm{C}$ allele was sparser $(27 \%)$ in the investigated pig populations with the highest frequency in the PL pig where CC genotype showed 0.43 frequency. In the Pietrain pigs, only one $\mathrm{CC}$ gilt was observed (Table S3). The PL and PLW populations were not consistent with HWE.

Table S3. The frequencies of alleles and genotypes of rs332398561 (C/A) of OBSL1 gene in 5 Polish breeds

\begin{tabular}{|c|c|c|c|c|c|c|}
\hline \multirow{3}{*}{ Pig breed } & \multicolumn{6}{|c|}{ Frequencies of rs332398561 (C/A) of OBSL1 } \\
\hline & \multicolumn{3}{|c|}{ Genotype } & \multicolumn{2}{|c|}{ Allele } & \multirow{2}{*}{$\begin{array}{l}\text { HWE* } \\
\text { (P-value) }\end{array}$} \\
\hline & $\mathrm{CC}$ & $\mathrm{AC}$ & AA & $\mathrm{C}$ & A & \\
\hline Polish Large White & $0.40(80)$ & $0.45(91)$ & $0.30(15)$ & 0.47 & 0.53 & 0.11 \\
\hline Polish Landrace & $0.11(14)$ & $0.57(69)$ & $0.32(39)$ & 0.39 & 0.61 & 0.04 \\
\hline Puławska & $0.50(66)$ & $0.38(50)$ & $0.11(15)$ & 0.70 & 0.30 & 0.25 \\
\hline Duroc & $0.84(48)$ & $0.14(8)$ & $0.02(1)$ & 0.91 & 0.09 & 0.35 \\
\hline Pietrain & $0.33(26)$ & $0.53(42)$ & $0.14(11)$ & 0.59 & 0.41 & 0.36 \\
\hline Total & $0.40(234)$ & $0.44(260)$ & $0.16(96)$ & 0.87 & 0.13 & \\
\hline
\end{tabular}

*HWE - Hardy-Weinberg Equilibrium: if P-value $<0.05$ - not consistent with HWE; not accurate if $<5$ individuals in any genotype group. In brackets number of individuals in genotype group.

rs344208061 mutation affected the IMF level. The CC Duroc pigs were characterised by $35 \%$ higher IMF levels $(\mathrm{P} \leq 0.01)$ than TT pigs. The opposite influence was observed in the Pietrain population, where TT gilts showed $43 \%$ higher IMF level $(\mathrm{P} \leq 0.01)$, their meat was more yellow $(\mathrm{P} \leq 0.05 \%)$, they had heavier loins $(900 \mathrm{~g}$, $\mathrm{P} \leq 0.01)$ and hams (400 g, trend), and lower DFI $(\mathrm{P} \leq 0.05)$ in comparison to heterozygous pigs. Moreover, TNS1 mutation affected LEA and FC that was significant in the PL breed where CC pigs showed $2.3 \mathrm{~cm}^{2}$ increased LEA and $120 \mathrm{~g}$ higher FC $(\mathrm{P} \leq 0.05)$ and increased loin mass was identified in CC Puławska pigs in comparison the other genotypes (Table 5).

\section{ENSSSCG00000033265 (lncRNA) gene}

The analyses of genotype and allele frequency of 31-bp-long indel (rs693217735) located in the second intron of ENSSSCG00000033265 gene showed that alleles without insertion were prevalent. However, in the Duroc pigs, this mutation occurs often; pigs with insertion in both alleles (genotype IN/IN) constituted $45 \%$ and this population was not consistent with HWE. 















The rs693217735 insertion significantly affected the IMF level in the Duroc and Polish Landrace pigs; IN/IN Duroc gilts showed over 50\% higher IMF level than DEL/DEL animals $(\mathrm{P} \leq 0.01)$, and in the PL breed, the differences were lower by $8 \%$. The higher IMF level values were identified in IN/IN pigs. In Puławska population, positive correlation between IN allele, daily gains and feed intake values was observed; IN/IN Puławska pigs consumed more forage (280 g/day) and grew faster (100 g/day) in comparison to DEL/DEL Puławska gilts (Table 6).

\section{TNP1 gene}

The rs 711919547 variation was localised upstream of the TNP1 gene, and an allele with insertion was frequent in Duroc and Polish Landrace, where IN/IN animals constituted $24 \%$ and $18 \%$, respectively. In the Pietrain population, IN/IN gilts were not observed.

The association analysis identified that rs 711919547 mutation affected fattening traits, where IN/IN gilts were characterised by increased daily gain, daily feed intake (PUL 310 g; PL - 150 g, Duroc - 230 g) thicker backfat thickness and higher IMF percentage (Table 7).

\section{Discussion}

The targeted enrichment DNA sequencing was used as a screening method to estimate the potential genetic markers for pig production traits in exceptionally QTLrich chromosome region (Piórkowska et al., 2018). However, the small animal group included in the previous study did not confirm the actual statistical effect on pig production traits. The present research attempted to establish the usefulness of the TEDNA-seq method as a tool for preselection; thus, a few polymorphisms recommended by this method were evaluated in association analysis. Moreover, the present study developed economical and easy-to-use methods to detect the chosen mutations.

The rs332253419 mutation changes the amino acid sequence of VIL1 gene, in the region encoding crucial protein domains, such as gelsolin-like (IPR007123) and villin/gelsolin (IPR007122). The gelsolin repeats participate in the regulation of the actin cytoskeleton because it binds to the barbed ends of actin filaments and caps them promoting nucleation by the assembly of monomers into filaments (Weeds et al., 1986). Moreover, gelsolin has a high affinity to fibronectin, and it interacts with gelsolin and tropomyosin modulating of actin dynamics (Khaitlina et al., 2013). In turn, villin is the actin-binding protein that can also prevent monomer exchange by end-blocking actin filaments (Northrop et al., 1986). Moreover, villin can protect against apoptosis induced by dextran sodium sulfate in the gastrointestinal epithelium. It probably regulates cell death by maintaining mitochondrial integrity (Tomar et al., 2009). Moreover, Mziaut et al. (2016) showed that VIL1 is related to type 1 diabetes mellitus along with two other genes located in the SSC15 region that were previously analysed for their effect on pig production traits such as $A B C A 12$ (Piórkowska et al., 2014), and IGFBP5. In the present study, the identified VIL1 mu- 
tation located in SSC15 QTL-rich region did not strongly affect pig phenotype. The weak effect of IMF content in the PLW and Duroc pigs did not positively correlate with an influence on meat content. Therefore, the reduction of $\mathrm{T}$ allele frequency in the examined pig populations would not be beneficial. The Ensembl genome browser provides information about numerous interesting mutations within the VIL1 gene, but they were not identified during TEDNA-seq analysis. Nevertheless, VIL1 plays an essential role in controlling the cell shape and the future research concerning the regulation of VIL1 expression and variation should be considered.

The OBSL1 mutation, which was identified by HRM, was previously included into Axiom Genotyping Array, Illumina_PorcineSNP60 and GGP Porcine HD chips. The present study emphasised its effect on a few important pig productive traits, including IMF level or backfat thickness. The obtained results suggest that the selection for the reduction of A allele would result in economic and meat quality benefits in the investigated pig population. However, this selection would be difficult in the PL population due to the dominance of A allele. On the other hand, obscurin-like-1 (OBSL1) protein is engaged in the complex with titin, a giant protein of M-band sarcomeres (Fukuzawa et al., 2008). OBSL1 interacts with myomesin, which is myosin cross-linking protein. Geisler et al. (2007) indicated that OBSL1 is a novel member of the UNc-89/obscurin family and is likely a cytoskeleton adaptor that plays a role in linking specific cytoskeletal elements with membrane-bound complexes within the cell membrane and nuclear envelope. Moreover, OBSL1 may participate in the scaffolding of signalling complexes to specific intracellular sites. OBSL1, like titin and other sarcomeric proteins, shares a common immunoglobulin (Ig)-rich modular structure (Fukuzawa et al., 2008). In humans, the mutation in the OBSL1 gene has been linked to the hereditary growth retardation associated with a 3-M syndrome where obscurin-like-1 is responsible for the regulation of CUL-7 level and IGFBPs (Huber et al., 2010). Therefore, OBSL1 acts as a regulator of Cul7-RING (FBXW8) ubiquitin-protein ligase, and is critical for the ubiquitin ligase pathway that regulates Golgi morphogenesis. In the present study, the identified OBSL1 mutation showed an interesting relationship with pig phenotype and the selection targeted for A allele seems to be promising. The role of obscurin-like-1 in the development and organisation of myofibril is still unclear, so research regarding its role in the expression of pig production traits should be continued.

Subsequently, the TNS1 missense mutation, although it does not change any important tensin domains, affected pig phenotype in the Duroc, PL and Pietrain populations. The TNS1 variant located in the first exon leads to exchange of alanine to threonine in amino acid sequence, and hydrophobic amino acid for a polar one, which prefers to be surrounded by water. Moreover, threonine hydroxyl group is fairly reactive and can form hydrogen bonds with a variety of polar substrates. Therefore, this change can significantly affect protein structure and function (Betts and Russell, 2003). Tensin 1, encoded by TNS1, is localised at the cell membrane (focal adhesions) in the region where the cell attaches to the extracellular matrix. Tensin binds to the barbed end of actin filaments and can cross-link them (Bianchi et al., 2006; Lo et al., 1994 a). Tensin contains an Src homology 2 (SH2) domain that interacts with PI3K and pl130Cas protein (Auger et al., 1996). The aggregation of integrin 
recruits tensin at the focal-adhesion site, which suggests that tensin plays a role as a structural protein and participates in signal transduction (Lo et al., 1994 b). Ishii and Lo (2001) examining the effect of tensin in skeletal-muscle regeneration using knockout tensin mice observed that they had defective satellite-cell activation and proliferation. Moreover, their skeletal muscle exhibited slower regeneration after the injury that could be associated with the perturbation of the integrin complex in promoting proliferation. The present study showed that the missense variant of the TNS1 was not inert in relation to the pig phenotype, which was particularly evident in the Duroc and Pietrain populations. It can be associated with TNS1 variant function during gene expression regulation or its linkage with another important causal mutation. Because tensin 1 plays a highly important role in cytoskeletal organization, cell-cell signal transduction and skeletal muscle regeneration, the TNS1 mutations should be considered in future pig marker research.

The present study also attempted to evaluate the effects on the pig phenotype of two insertions localised in the QTL-rich SSC15 that may have gene expression regulatory function. The first 16-bp-long is located in the intergenic region between TNP1 gene and miscRNA element. This locus contains over 60 identified QTLs associated with pork quality, growth traits, fat level and loin mass (pig QTLs database). The present study found an association of this mutation mainly with fattening characteristics such as feed conversion, daily feed intake and daily gain. The rs711919547 insertion affected backfat thickness, meat yellowness and IMF content in pigs. RegRNA 2.0 software (Chang et al., 2013) indicated that this insertion adds one exon silencer motif named fibronectin eda exon, one splice acceptor, and removes the UTRsite motif named Musashi binding element (MBE). The regulatory function of this sequence is unknown, but its frequency probably shifted with selection, as was observed in particular pig populations with different selection directions. Additionally, the rs711919547 insertion is localised in the neighbourhood of TNP1 gene that encodes a transition protein 1 previously investigated mainly regarding sperm maturation and male fertility (Aoki et al., 2006). In turn, miscRNA elements are short miscellaneous RNAs that have a variety of functions, such as catalytic during RNA processing and can serve as switches or gene silencers (RNAi) tagging mRNA for destruction (Vaishnaw et al., 2010). The role of rs711919547 mutation is still unknown, but its sensitivity to the selection process indicates the relation to selected pig traits.

The last examined mutation located within the first intron of ENSSS$C G 00000033265$ belongs to the long non-coding RNA family. The lncRNAs play a regulatory role in the gene expression process. They target transcriptional activators or repressors (Feng et al., 2006) because they often contain the ultraconservative gene elements that contribute gene inactivation, leading to frequent apoptosis and favouring tumorigenesis (Calin et al., 2007). Moreover, they control post-transcription mRNA processing by pairing with target mRNAs, similarly to miRNAs, masking key mRNA processing elements (Beltran et al., 2008). In the present study, the obtained results indicate that ENSSSCG00000033265 insertion was involved in the controlling of the IMF level, which was mainly observed in Duroc and PL pigs, as well as in the regulation of feed intake. This mutation could be related to gene 
expression regulation, because it changes the binding site for over 50 transcription factors such as MYOD, PAX-8, PAX-6, LIM1, MYF3 and TFIIB, and adds one motif for the binding exonic splicing enhancer SC35 which was identified by RegRNA 2.0 software.

\section{Conclusion}

In summary, the OBSL1 mutation seems to be the most promising potential DNA marker that can be used for selection purposes and should not be omitted in lowdensity SNP microarrays. However, the usefulness of the proposed marker should be control in extended association study including other pig populations. Besides, the TEDNA-seq method can be used as a pre-selective method for identifying molecular markers, but with an accompanying association study including a large number of animals.

\section{Conflict of interests}

The authors declare no conflicts of interest.

\section{Acknowledgements}

The research will be performed on biological material derived from pigs maintained and slaughtered in the Test Pig Station (National Research Institute of Animal Production). In the Test Station pigs are slaughtered, dissected and after carcass evaluation, meat is standard intended for consumption. Therefore, our research does not require the approval of the Animal Experimentation Committee.

\section{References}

A o k i V.W., Christen se n G.L., A t k in s J.F., C a r r e 11 D.T. (2006). Identification of novel polymorphisms in the nuclear protein genes and their relationship with human sperm protamine deficiency and severe male infertility. Fertil. Steril., 86: 1416-1422.

A uger K.R., Songyang Z., Lo S.H., Roberts T.M., Chen L.B. (1996). Platelet-derived growth factor-induced formation of tensin and phosphoinositide 3-kinase complexes. J. Biol. Chem., 271: 23452-23457.

Beltran M., Puig I., Peña C., García J.M., Álvarez A.B., Peña R., Bonilla F., De Herrero s A.G. (2008). A natural antisense transcript regulates Zeb2/Sip1 gene expression during Snail1-induced epithelial-mesenchymal transition. Genes Dev., 22: 756-769.

B e t t s M.J., R u s s e 11 R.B. (2003). Amino acid properties and consequences of subsitutions. In: Bioinformatics for Geneticists. Wiley, 4: 311-342.

B i a n chi M., P etra c c i M., Fran ch in i A., C a v a n i C. (2006). The occurrence of deep pectoral myopathy in roaster chickens. Poultry Sci., 85: 1843-1846.

Cal in G.A., Li u C.G. Ferracin M., Hys 1 op T., S pizzo R., S evignani C., Fabbri M., Cimmino A., Le e E.J., Wojcik S.E., Shimizu M., Tili E., Rossi S., Taccioli C., Pichiorri F., Liu X., Zupo S., Herlea V., Gramantieri L., Lanza G., Alder H., Rassenti L., Volinia S., S chmittgen T.D.D., Kipps T.J., Negrini M., Croce C.M. (2007). Ultraconserved regions encoding ncRNAs are altered in human leukemias and carcinomas. Cancer Cell, 12: 215-229.

Chang T.H., Huang H.Y., Hsu J.B.K., Weng S.L., Horng J.T., Huang H.D. (2013). An enhanced computational platform for investigating the roles of regulatory RNA and for identifying functional RNA motifs. BMC Bioinformatics, 14. 
Dall'Olio S., Scotti E., Costa L.N., Fontanesi L. (2018). Effects of single nucleotide polymorphisms and haplotypes of the protein kinase AMP-activated non-catalytic subunit gamma 3 (PRKAG3) gene on production, meat quality and carcass traits in Italian Large White pigs. Meat Sci., 136: 44-49.

D odds W., Whiles M., D odd s W.K., Whiles M.R. (2010). Nitrogen, sulfur, phosphorus, and other nutrients. Elsevier Inc., Amsterdam, The Netherlands.

F eng J., Bi C., C lark B.S., M a d y R., Shah P., K o htz J.D. (2006). The Evf-2 noncoding RNA is transcribed from the Dlx-5/6 ultraconserved region and functions as a Dlx-2 transcriptional coactivator. Genes Dev., 20: 1470-1484.

Fukuzawa A., Lange S., Holt M., Vihola A., Carmignac V., Ferreiro A., Udd B., G a u t e 1 M. (2008). Interactions with titin and myomesin target obscurin and obscurin-like 1 to the M-band - Implications for hereditary myopathies. J. Cell Sci., 121: 1841-1851.

G e i s 1 e r S.B., R o b i n s o n D., H a u ring a M., R a e k e r M.O., B or is o v A.B., We s t fall M. V., Rus s e 11 M.W. (2007). Obscurin-like 1, OBSL1, is a novel cytoskeletal protein related to obscurin. Genomics, 89: 521-531.

Huber C., Fradin M., Edouard T., Le Merrer M., Alanay Y., Da Silva D.B., David A., Hamamy H., Van Hest L., Lund A.M., Michaud J., Oley C., Patel C., Rajab A., Skidmore D.L., Stewart H., Tauber M., Munnich A., Cormier-Dai $\mathrm{re} \mathrm{V.} \mathrm{(2010).} \mathrm{OBSL1} \mathrm{mutations} \mathrm{in} \mathrm{3-M} \mathrm{syndrome} \mathrm{are} \mathrm{associated} \mathrm{with} \mathrm{a} \mathrm{modulation} \mathrm{of} \mathrm{IGFBP2} \mathrm{and}$ IGFBP5 expression levels. Hum. Mutat., 31: 20-26.

I s h i i A., L o S.H. (2001). A role of tensin in skeletal-muscle regeneration. Biochem. J., 356: 737-745.

Ji J., Yan G., Chen D., Xia o S., Ga o J., Zhang Z. (2019). An association study using imputed whole-genome sequence data identifies novel significant loci for growth-related traits in a Duroc $\times$ Erhualian F 2 population. J. Anim. Breed. Genet., 136: 217-228.

$\mathrm{K}$ ha it lin a S., Fitz H., H in s s en H. (2013). The interaction of gelsolin with tropomyosin modulates actin dynamics. FEBS J., 280: 4600-4611.

L o S.H., J a n m e y P.A., H a r tw i g J.H., C h e n L.B. (1994 a). Interactions of tensin with actin and identification of its three distinct actin-binding domains. J. Cell Biol., 125: 1067-1075.

L o S.H., We is b e rg E., C h e n L.B. (1994 b). Tensin: A potential link between the cytoskeleton and signal transduction. BioEssays.

Messeguer X., Escudero R., Farré D., Núñez O., Martínez J., Albà M.M. (2002). PROMO: Detection of known transcription regulatory elements using species-tailored searches. Bioinformatics, 18: 333-334.

Mziaut H., Mulligan B., Hoboth P., Otto O., Ivanova A., Herbig M., Schumann D., Hildebrandt T., Dehghany J., Sönmez A., Münster C., Me yer-Hermann M., Guck J., Kalaidzidis Y., Solimena M. (2016). The F-actin modifier villin regulates insulin granule dynamics and exocytosis downstream of islet cell autoantigen 512. Mol. Metab., 5: 656-668.

Northrop J., Weber A., Mooseker M.S., Franzini-Armstrong C., Bishop M.F., D u by a k G.R., Tu cker M., Wa ls h T.P. (1986). Different calcium dependence of the capping and cutting activities of villin. J. Biol. Chem., 261: 9274-9281.

Piórkowska K., Ropka-Molik K., Szmatoła T., Zygmunt K., Tyra M. (2014). Association of a new mobile element in predicted promoter region of ATP-binding cassette transporter 12 gene (ABCA12) with pig production traits. Livest. Sci., 168: 38-44.

P iórkow s k a K., Ty ra M., R o pka-Molik K., Podbiels k a A. (2017). Evolution of peroxisomal trans-2-enoyl-CoA reductase (PECR) as candidate gene for meat quality. Livest. Sci., 201: 85-91.

Piórkowska K., Żukowski K., Ropka-Molik K., Tyra M. (2018). Deep sequencing of a QTL-rich region spanning 128-136 Mbp of pig chromosome 15. Gene, 647: 268-275.

Ponsuksili S., Trako oljul N., Ha d li ch F., Ha a ck F., Murani E., Wimmers K. (2017). Genetic architecture and regulatory impact on hepatic microRNA expression linked to immune and metabolic traits. Open Biol., 7.

Ropka-Molik K., Dusik A., Piórkowska K., Tyra M., Oczkowicz M., Szmato1 a T. (2015). Polymorphisms of the membrane-associated ring finger 4, ubiquitin protein ligase gene (MARCH4) and its relationship with porcine production traits. Livest. Sci., 178: 18-26. 
R ó ży c k i M., Ty r a M. (2010). Methodology for the evaluation of fattening and slaughter value of pigs carried out at the Polish Pig Testing Station (SKURTCh). Report on pig breeding in Poland (in Polish). IZ PIB, XXVIII: 93-117.

S oxhlet F. (1879). Soxhlet, über gewichtsanalytische Bestimmung des Milchfettes. Polytech. J., 232.

Stuczyńska A., P iórkowska K., Tyra M., Żukowski K. (2018). The effect of QTL-rich region polymorphisms identified by targeted DNA-seq on pig production traits. Mol. Biol. Rep., 45: 361-371.

To m ar A., Ge org e S.P., M a thew S., Kh u ran a S. (2009). Differential effects of lysophosphatidic acid and phosphatidylinositol 4,5-bisphosphate on actin dynamics by direct association with the actin-binding protein villin. J. Biol. Chem., 284: 35278-35282.

Tyra M., Żak G. (2013). Analysis of the possibility of improving the indicators of pork quality through selection with particular consideration of intramuscular fat (imf) content. Ann. Anim. Sci., 13: $33-44$.

Vaishnaw A.K., Gollob J., Gamba-Vitalo C., Hutabarat R., Sah D., Meyers R., de Fougerolles T., Marag a nore J. (2010). A status report on RNAi therapeutics. Silence, 1: 14 .

We e d s A.G., G o o ch J., P ope B., Harris H.E. (1986). Preparation and characterization of pig plasma and platelet gelsolins. Eur. J. Biochem., 161: 69-76.

Zh ang C., Wang Z., B ruce H., Ke mp R.A., Charagu P., Mi ar Y., Yang T., P las tow G. (2015). Genome-wide association studies (GWAS) identify a QTL close to PRKAG3 affecting meat $\mathrm{pH}$ and colour in crossbred commercial pigs. BMC Genet., 16.

Received: 30 IX 2019

Accepted: 9 XII 2019 